\title{
Organocatalytic Enantioselective Conjugate Addition to Alkynones
}

Marco Bella and Karl Anker Jørgensen*

Danish National Research Foundation: Center for Catalysis, Department of Chemistry, Aarhus University, DK-8000 Aarhus C, Denmark

\section{Supporting Information}

General Methods. The ${ }^{1} \mathrm{H}$ NMR, ${ }^{13} \mathrm{C}$ NMR and ${ }^{19} \mathrm{~F}$ NMR spectra were recorded at 400 $\mathrm{MHz}, 100 \mathrm{MHz}$ and $376 \mathrm{MHz}$, respectively. The chemical shifts are reported in ppm downfield to TMS $(\delta=0)$ for ${ }^{1} \mathrm{H}$ NMR $(\delta=77.5)$ for ${ }^{13} \mathrm{C}$ NMR relative to the central $\mathrm{CDCl}_{3}$ resonance. Flash chromatography (FC) was carried out using Merck silica gel 60 (230-400 mesh). Optical rotations were measured on a Perkin-Elmer 241 polarimeter. The enantiomeric excess (ee) of the products was determined by HPLC using a Daicel Chiralpak AS, AD, and Chiralcel OJ, OD columns (wavelength $=254 \mathrm{~nm}$ ) with $i$ $\mathrm{PrOH} /$ hexane as the eluent or by chiral GC using a Chrompack CP-Chirasil Dex C $\beta$ or Astec G-TA columns.

Materials. Alkynones $1 \mathrm{~b},{ }^{1} \mathbf{1 c}, \mathbf{1 d},{ }^{2} \mathbf{1 e},{ }^{3} \mathbf{1 f}, \mathbf{1 g}$ and $\mathbf{1 h ^ { 2 }}$ were all prepared according to literature procedure. ${ }^{3} \beta$-Diketones $\mathbf{2 a}, \mathbf{2 b}, \mathbf{2 e}$, alkynone 1a, [DHQ] ${ }_{2} \mathrm{PHAL},[\mathrm{DHQ}]_{2} \mathrm{AQN}$, $[\mathrm{DHQ}]_{2} \mathrm{PYR},[\mathrm{DHQD}]_{2} \mathrm{PHAL}$ quinine, cinchonine, (S)-(+)-1-(2-pyrrolidinylmethyl)pyrrolidine, $\mathrm{Et}_{3} \mathrm{~N}, \mathrm{Bu}_{3} \mathrm{P}$, toluene, were obtained from Aldrich and used as received. $O$-Ac cinchonine, $\beta$-diketones $\mathbf{2 c}, \mathbf{2 d}, \mathbf{2 e}, \mathbf{2 f}$ and $\mathbf{2 g}$ were preparing according to standard literature procedures.

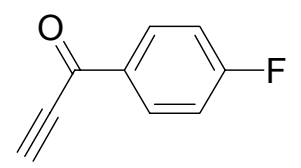

1c: Yield: $82 \% ;{ }^{1} \mathrm{H}$ NMR $\delta 8.13(\mathrm{dd}, 2 \mathrm{H}, J(H) 8.8 \mathrm{~Hz}, J(F) 5.2 \mathrm{~Hz}), 7.12(\mathrm{dd}, 2 \mathrm{H}, J(H)$ $8.8 \mathrm{~Hz}, J(F) 5.2 \mathrm{~Hz}), 3.4(\mathrm{~d}, 1 \mathrm{H}, J(F) 0.8 \mathrm{~Hz}) ;{ }^{13} \mathrm{C} \mathrm{NMR} \delta 176.24,167.15(\mathrm{~d}, J(F) 257.2$ $\mathrm{Hz}), 133.10(\mathrm{~d}, J(F) 2.3 \mathrm{~Hz}), 132.90$ (d, $J(F) 10.0 \mathrm{~Hz}), 116.46(\mathrm{~d}, J(F) 22.0 \mathrm{~Hz}), 82.43$, $81.32 ;{ }^{19} \mathrm{~F}$ NMR $\delta-102.7(\mathrm{~m})$. 


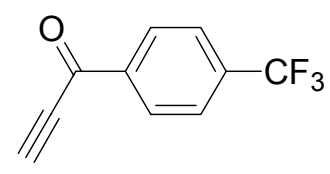

1f: Yield: $85 \%$; ${ }^{1} \mathrm{H}$ NMR $\delta 8.25$ (d, 2H, $\left.J 8.4 \mathrm{~Hz}\right), 7.78$ (d, 2H, $\left.J 8.4 \mathrm{~Hz}\right), 3.56$ (s, $\left.1 \mathrm{H}\right)$; ${ }^{13} \mathrm{C}$ NMR $\delta 176.65,138.94,135.96(\mathrm{q}, J(F) 33.0 \mathrm{~Hz}), 130.39,126.21$ (q, $J(F) 3.8 \mathrm{~Hz}$ ), $123.86\left(\mathrm{q}, J(F) 272.4 \mathrm{~Hz}\right.$ ), 82.60, 80.20; ${ }^{19} \mathrm{~F}$ NMR $\delta-63.7$ (s).

0

1h: Yield: $83 \%$; ${ }^{1} \mathrm{H}$ NMR $\delta$ 7.4-7.2 (m, 5H), 3.28 (s, $\left.1 \mathrm{H}\right), 3.1-2.9(\mathrm{~m}, 4 \mathrm{H}) ;{ }^{13} \mathrm{C}$ NMR $\delta$ $186.75,140.39,129.06,128.80,126.85,81.76,79.34,47.41,30.01$.

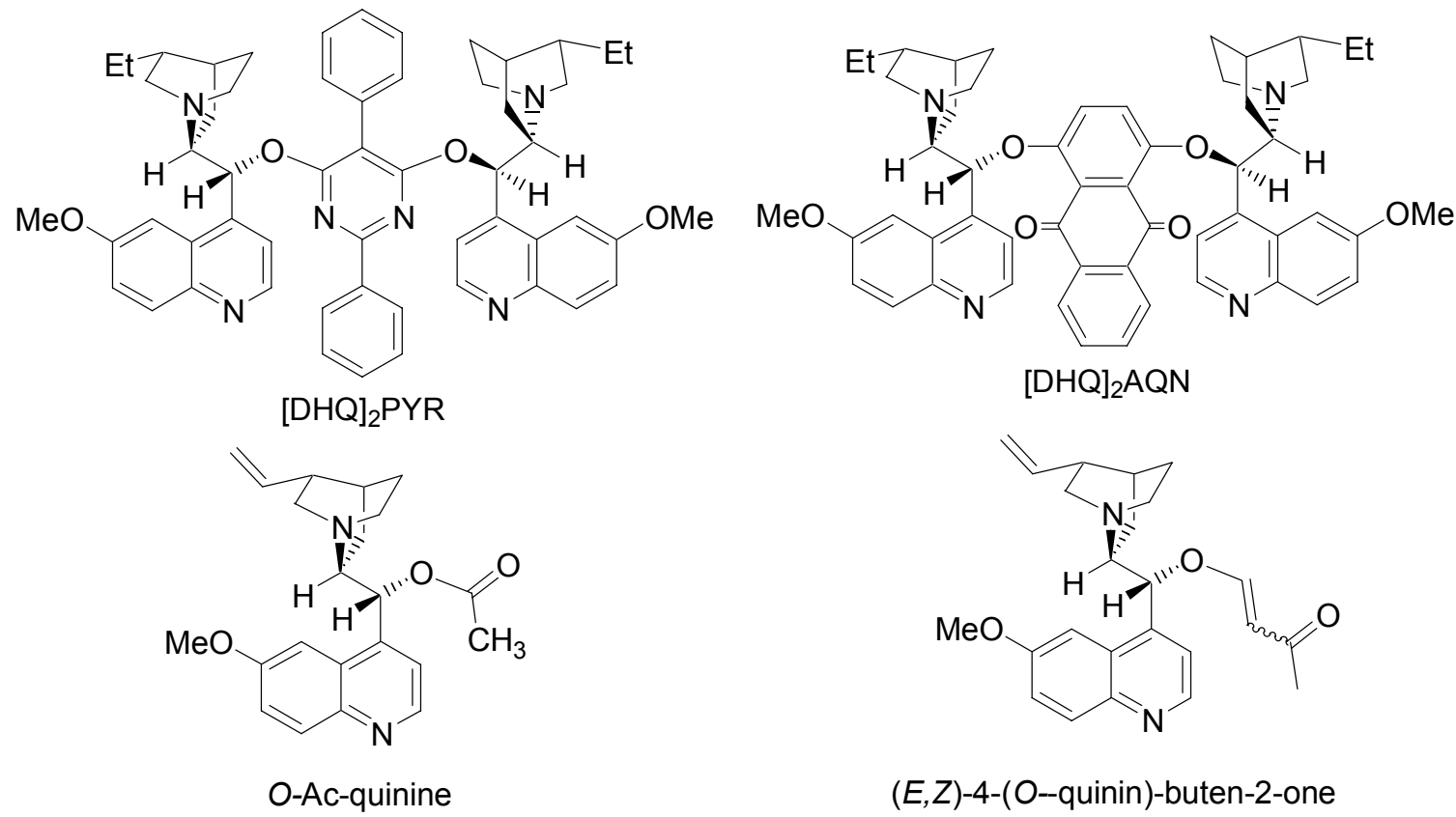

Figure 1: Structure of some of the cinchona alkaloids derivatives tested.

Screening of reaction conditions for the conjugate addition of butyn-2-one 1 a to acetyl cyclopentanone 2 a (Table 1$)$.

The cinchona-base ( $5 \mathrm{~mol} \%, 0.005 \mathrm{mmole})$ was placed a in a test tube and acetyl cyclopentanone 2a (12.6 mg, $0.1 \mathrm{mmole}$ ), dissolved in $2 \mathrm{~mL}$ of toluene, was added. Butyn-2-one 1a (10 mg, $0.116 \mathrm{mmole}$ ) was added via syringe and the resulting solution allowed to stir at room temperature until all the 2a has reacted $(0.5-4 \mathrm{~h}) . \mathrm{Et}_{2} \mathrm{O}(10 \mathrm{~mL})$ 
was added to the solution and filtered through a plug of silica gel $(0.5 \mathrm{~g})$ to be directly analyzed by CSP-GC.

A larger scale preparation (400 mg acetyl cyclopentanone 2a, $317 \mathrm{mg}$ 2-butynone 1a and $123 \mathrm{mg}$ [DHQ] $]_{2} \mathrm{PHAL}$ in $63 \mathrm{~mL}$ of toluene) was purified by FC. The solution was loaded on a column packed with $20 \mathrm{~g}$ of silica gel, toluene was removed, and then the product was separated eluting, first with pentane, followed by pentane/Et ${ }_{2} \mathrm{O} 6: 1 ; 220 \mathrm{mg}$ of (Z)-3a (33\% yield) and $438 \mathrm{mg}$ of $(E)-3 \mathbf{a}(64 \%$ yield) were obtained as transparent oils.

\section{General procedure for the isomerization of alkynones double bond.}

The crude mixture of addition products $(Z)$ - and $(E)-3 \mathbf{a}(0.4 \mathrm{mmole}, 83 \mathrm{mg}, 2: 1$ ratio $(Z) /(E)$, ee $=40 \%(Z)$, ee $=70 \%(E))$ was dissolved in $8 \mathrm{~mL}$ of toluene and $8 \mathrm{mg}$ of $\mathrm{Bu}_{3} \mathrm{P}$ $(0.04 \mathrm{mmol}, 10 \% \mathrm{~mol})$ was added. The solution was immediately placed under vacuum in a hot water bath warmed up to be $40{ }^{\circ} \mathrm{C}$ and the solvent was removed by evaporation over $5 \mathrm{~min}$. The resulting dark-yellowish oil was first analyzed by ${ }^{1} \mathrm{H}$ NMR and the $(E)$ - and (Z)-isomer ratio was found to be $>50: 1$, followed by purification by FC (first pentane, then pentane/ $\mathrm{Et}_{2} \mathrm{O} 6: 1$ to afford $67 \mathrm{mg}(81 \%$ yield $)$ of pure $(E)-3 \mathbf{a}(60 \%$ ee).

3a: The ee was determined by HPLC using Chiralpak AD column (hexane $i$-PrOH (85:15); flow rate $1.0 \mathrm{~mL} / \mathrm{min} ;(Z)-3 \mathbf{a}: \tau_{\text {minor }}=9.4 \mathrm{~min} ; \tau_{\text {major }}=11.0 \mathrm{~min}$; $(E)-3 \mathbf{a}: \tau_{\text {minor }}=$ $12.5 \mathrm{~min} ; \tau_{\text {major }}=13.6 \mathrm{~min}$ ) and by GC using a chiral Astec G-TA column: rate 10 ${ }^{\circ} \mathrm{C} / \mathrm{min}$, starting $\mathrm{T}=70^{\circ} \mathrm{C}$; $\mathrm{T}$ final $180{ }^{\circ} \mathrm{C}$ for $10 \mathrm{~min}$; $(Z)-3 \mathbf{a}: \tau_{\text {major }}=14.1 \mathrm{~min}, \tau_{\text {minor }}=$ $14.4 \mathrm{~min} ;(E)-3 \mathrm{a}: \tau_{\text {major }}=15.4 \mathrm{~min}, \tau_{\text {minor }}=15.5 \mathrm{~min}$.

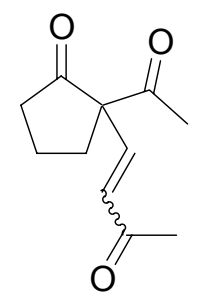

(E)-3a: ${ }^{1} \mathrm{H}$ NMR $\delta 6.98$ (d, 1H, $\left.J 16.4 \mathrm{~Hz}\right), 6.09$ (d, 1H, $\left.J 16.4 \mathrm{~Hz}\right), 2.8-2.7$ (m, 1H), 2.4$2.2(\mathrm{~m}, 2 \mathrm{H}), 2.25,(\mathrm{~s}, 3 \mathrm{H}), 2.18(\mathrm{~s}, 3 \mathrm{H}), 2.30-2.20(\mathrm{~m}, 2 \mathrm{H}), 2.13(\mathrm{~s}, 3 \mathrm{H}), 2.0-1.8(\mathrm{~m}, 3 \mathrm{H})$; ${ }^{13} \mathrm{C}$ NMR $\delta 212.46,200.35,197.98,143.15,132.21,71.92,38.33,31.51,27.89,27.55$, 
$19.79 ;[\alpha]^{\mathrm{rt}}=+18.2\left(c=1.51 \mathrm{~g} / 100 \mathrm{~mL}, \mathrm{CHCl}_{3}, 70 \%\right.$ ee - reaction performed at room temperature).

(Z)-3a: ${ }^{1} \mathrm{H}$ NMR $\delta 6.33(\mathrm{~d}, 1 \mathrm{H}, J 11.2 \mathrm{~Hz}), 6.30$ (d, 1H, $\left.J 11.2 \mathrm{~Hz}\right), 2.95-2.85(\mathrm{~m}, 1 \mathrm{H})$, 2.4-2.3 (m, 2H), 2.19, (s, 3H), 2.10 (s, 3H), 2.1-1.6 (m, 3H); ${ }^{13} \mathrm{C}$ NMR 213.90, 200.76, $189.51,143.58,129.38,71.93,38.05,33.91,31.51,27.44,20.15 ;[\alpha]^{\mathrm{rt}}=+59.6(c=2.97$ $\mathrm{g} / 100 \mathrm{~mL}, \mathrm{CHCl}_{3}, 40 \%$ ee - reaction performed at room temperature); HRMS calc. (mixture of isomers) $\mathrm{C}_{11} \mathrm{H}_{14} \mathrm{NaO}_{3}: 217.08406$; found: 217.0840 .

Reaction of compound $(\boldsymbol{Z} / \boldsymbol{E})$-3a with silica gel. Compound 3a, $66 \mathrm{mg} 1: 2 \mathrm{ratio} Z / E$, ee $=40 \%(Z)$, ee $=70 \%(E)$, was dissolved in $2 \mathrm{~mL}$ of $\mathrm{CH}_{2} \mathrm{Cl}_{2}$ and the solution adsorbed $3 \mathrm{~g}$ of silica gel. The mixture was dried by rotary evaporator. After $6 \mathrm{~h}$ the silica was washed with $\mathrm{Et}_{2} \mathrm{O}(100 \mathrm{~mL})$, the solvent evaporated to afford a yellowish dense liquid. Purification by FC (pentane, then pentane: $\mathrm{Et}_{2} \mathrm{O}$ 6:1) afforded $15 \mathrm{mg}$ of compound 3a as only the $(Z$ )-isomer (yield $=68 \%$ based on only $(Z)-3 a$, ee $=40 \%$ ) and $23 \mathrm{mg}$ of compound $(E) \mathbf{4} \mathbf{a}^{4}(52 \%$ yield based on only $(E)-3 \mathbf{a})$.

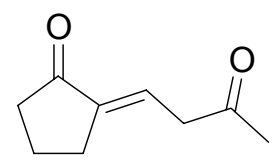

(E)-4a: ${ }^{1} \mathrm{H}$ NMR $\delta$ 6.65-6.55 (m, 1H), 3.22 (d, 2H $\left.J 7.2 \mathrm{~Hz}\right), 2.6-2.5(\mathrm{~m}, 2 \mathrm{H}), 2.29(\mathrm{t}, 2 \mathrm{H}$ $J$ 8.0 Hz) 2.14, (s, 3H), 1.95.1-1.85 (m, 2H); ${ }^{13} \mathrm{C}$ NMR 206.85, 204.78, 140.58, 126.64, 44.76, 38.92, 30.43, 27.34, 20.03; HRMS calc. $\mathrm{C}_{9} \mathrm{H}_{12} \mathrm{NaO}_{2}$ : 175.0735; found: 175.0734.

\section{General procedure for the catalytic asymmetric Michael addition to aryl alkynones.}

$\beta$-Diketones (Table 2) (0.3 mmole) and [DHQ] $]_{2}$ PHAL (0.015 mmol, $5 \mathrm{~mol} \%$ catalyst loading) were dissolved in toluene $(8 \mathrm{~mL})$ and the solution was cooled to $-20{ }^{\circ} \mathrm{C}$. Alkynones (see Table 1 and 2) were dissolved in $4 \mathrm{~mL}$ of toluene, the solution cooled to $-20^{\circ} \mathrm{C}$ and added drop-wise to the $\beta$-diketone solution. The reaction mixture was stirred at $-20^{\circ} \mathrm{C}$ for $24 \mathrm{~h}, \mathrm{Et}_{2} \mathrm{O}(5 \mathrm{~mL})$ was added and the solution filtered over a plug (a Pasteur pipette which the end was filled with cotton wool and loaded with $0.5 \mathrm{~g}$ of silica gel). The silica was washed with additional $2 \mathrm{~mL}$ of $\mathrm{Et}_{2} \mathrm{O}$. The organic solvents were evaporated to 
give pure compounds, all as pale yellow dense oils, with yields and enantiomeric excesses as reported in Table 2 .

General procedure for tandem addition to alkynones-isomerization of the double bond. After the catalytic addition reaction to aryl alkynones has been stirred for $24 \mathrm{~h}$, (see above procedure) the solution was transferred in a round-bottomed flask, $\mathrm{Bu}_{3} \mathrm{P}(0.03$ mmoles, $10 \%$ ) was added and the flask immediately put under vacuum and solvent removed at $40{ }^{\circ} \mathrm{C}$. The resulting dark-yellow oils were purified by $\mathrm{FC}$ (note: the compounds are slightly unstable on silica) to afford only the $(E)$ isomeric products as reported in Table 2.

3b: The ee was determined by HPLC using Chiralpak AS+AD columns (hexane $/ i-\mathrm{PrOH}$ (90:10); flow rate $1.0 \mathrm{~mL} / \mathrm{min}$; $(Z)-3 \mathbf{b}: \tau_{\text {minor }}=20.2 \mathrm{~min}, \tau_{\text {major }}=22.5 \mathrm{~min} ;(E)-\mathbf{3 b}: \tau_{\text {minor }}$ $\left.=21.1 \mathrm{~min}, \tau_{\text {major }}=24.6 \mathrm{~min}\right)$.

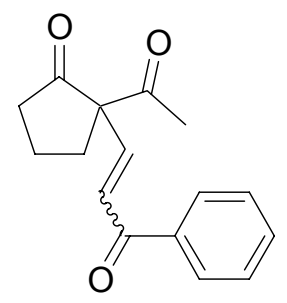

Yield: $99 \%$

(E)-3b: ${ }^{1} \mathrm{H}$ NMR $\delta$ 7.85-7.80 (m, 2H), 7.60-7.55 (m, 1H), 7.50-7.45 (m, 2H), 7.14 (d, 1H, $J 16.0 \mathrm{~Hz}$ ), 6.94 (d, 1H, $J 16.0 \mathrm{~Hz}$ ), 2.80 (ddd, 1H, $J$ 12.0, 6.8, $6.0 \mathrm{~Hz}$ ), 2.3-2.2 (m, 2H), $2.20(\mathrm{~s}, 3 \mathrm{H}), 2.05-1.95(\mathrm{~m}, 2 \mathrm{H}), 1.95-1.85(\mathrm{~m}, 1 \mathrm{H}) ;{ }^{13} \mathrm{C}$ NMR $\delta$ 212.37, 200.67, 189.89, $144.19,133.65,129.10,128.97,128.85,127.31,72.09,38.43,31.92,27.76,19.81 ;[\alpha]^{\mathrm{rt}}$ $=-13.2\left(c=1.25 \mathrm{~g} / 100 \mathrm{~mL}, \mathrm{CHCl}_{3}, 80 \%\right.$ ee - reaction performed at room temperature $)$.

(Z)-3b: ${ }^{1} \mathrm{H}$ NMR $\delta$ 7.85-7.80 (m, 2H), 7.60-7.55 (m, 1H), 7.50-7.45 (m, 2H), $6.97(\mathrm{~d}, 1 \mathrm{H}$, $J 12.0 \mathrm{~Hz}), 6.68(\mathrm{~d}, 1 \mathrm{H}, J 12.0 \mathrm{~Hz}), 3.05-2.95(\mathrm{~m}, 1 \mathrm{H}), 2.35-2.25(\mathrm{~m}, 2 \mathrm{H}), 2.26(\mathrm{~s}, 3 \mathrm{H})$, 2.1-1.8 (m, 3H); ${ }^{13} \mathrm{C}$ NMR $\delta 214.17,200.69,191.17,145.00,137.42,133.87,129.10$, 128.86, 127.06, 72.13, 37.93, 33.67, 27.63, 20.10; HRMS calc.: $\mathrm{C}_{16} \mathrm{H}_{16} \mathrm{NaO}_{3}$ 279.0997; found: 279.1006 . 
3c: The ee was determined by HPLC using Chiralpak AS+AD columns (hexane $/ i-\mathrm{PrOH}$ (85:15); flow rate $1.0 \mathrm{~mL} / \mathrm{min} ;(E)-3 \mathbf{c}: \tau_{\operatorname{minor}}=19.5 \mathrm{~min}, \tau_{\text {major }}=20.9 \mathrm{~min} ;(Z)-3 \mathbf{c}: \tau_{\operatorname{minor}}=$ $\left.26.5 \mathrm{~min}, \tau_{\text {major }}=46.9 \mathrm{~min}\right)$.

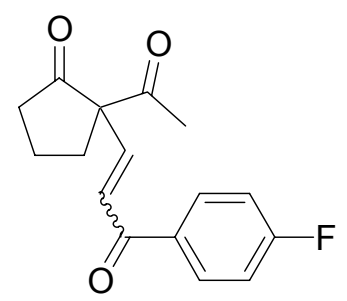

Yield: 99\%

(E)-3c: ${ }^{1} \mathrm{H}$ NMR $\delta 7.96(\mathrm{dd}, 2 \mathrm{H}, J(H) 8.8 \mathrm{~Hz}, J(F) 5.6 \mathrm{~Hz}), 7.23(\mathrm{~d}, 1 \mathrm{H}, 15.6 \mathrm{~Hz}), 7.16$ (dd, 2H, $J(H) 8.8 \mathrm{~Hz}, J(F) 5.6 \mathrm{~Hz}$ ), 7.00 (d, $1 \mathrm{H}, J 15.6 \mathrm{~Hz}$ ), 2.90 (ddd, 1H, $J$ 12.0, 6.8, $6.0 \mathrm{~Hz}$ ), 2.35-2.25 (m, 2H), 2.29 (s, 3H), 2.1-2.0 (m, 3H); ${ }^{13} \mathrm{C}$ NMR $\delta 212.37,200.60$, 188.23, 165.97 (d, $J(F) 255.6 \mathrm{~Hz}), 144.40,133.95$ (d, $J(F) 3.0 \mathrm{~Hz}), 131.68$ (d, $J(F) 9.8$ $\mathrm{Hz}), 126.93,116.29,(\mathrm{~d}, J(F) 22.0 \mathrm{~Hz}), 72.11,38.53,32.03,27.77,19.86 ;{ }^{19} \mathrm{~F}$ NMR $\delta$ $104.9(\mathrm{~m}) ;[\alpha]_{\mathrm{D}}^{\mathrm{tt}}=-18.5\left(c=1.70 \mathrm{~g} / 100 \mathrm{~mL}, \mathrm{CHCl}_{3}, 80 \%\right.$ ee - reaction performed at room temperature).

(Z)-3c: ${ }^{1} \mathrm{H}$ NMR $\delta 7.96(\mathrm{dd}, 2 \mathrm{H}, J(H) 8.8 \mathrm{~Hz}, J(F) 5.6 \mathrm{~Hz}), 7.16(\mathrm{dd}, 2 \mathrm{H}, J(H) 8.8 \mathrm{~Hz}$, $J(F) 5.6 \mathrm{~Hz}), 7.13(\mathrm{~d}, 1 \mathrm{H}, J 11.6 \mathrm{~Hz}), 6.69$ (d, 1H, $J 11.6 \mathrm{~Hz}), 3.0-2.9$ (m, 1H), 2.35-2.25 (m, 2H), 2.19 (s, 3H), 2.1-2.0 (m, 3H); ${ }^{13} \mathrm{C}$ NMR $\delta$ 214.07, 200.49, 189.64, 166.03 (d, $J(F) 255.4 \mathrm{~Hz}), 145.21,134.05$ (d, $J(F) 3.0 \mathrm{~Hz}), 131.71$ (d, $J(F) 9.8 \mathrm{~Hz}), 126.96,116.20$, (d, $J(F) 22.0 \mathrm{~Hz}), 72.15,37.93,33.72,27.59,20.01 ;{ }^{19} \mathrm{~F}$ NMR $\delta$-104.9. HRMS calc. : $\mathrm{C}_{16} \mathrm{H}_{15} \mathrm{FNaO}_{3}$ : 297.0902; found : 297.0895 .

3d: The ee was determined by HPLC using Chiralpak AS+AD columns (hexane $/ i-\mathrm{PrOH}$ (90:10); flow rate $1.0 \mathrm{~mL} / \mathrm{min} ;(E)-\mathbf{3 d}: \tau_{\text {minor }}=23.7 \mathrm{~min}, \tau_{\text {major }}=25.8 \mathrm{~min} ;(Z)-3 \mathbf{d}: \tau_{\text {minor }}$ $\left.=45.7 \mathrm{~min}, \tau_{\text {major }}=122.9 \mathrm{~min}\right)$. 


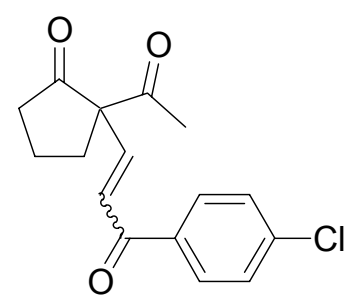

Yield: 99\%

(E)-3d: ${ }^{1} \mathrm{H}$ NMR $\delta 7.80$ (d, 2H, J 8.8 Hz), 7.38 (d, 2H, J 8.8 Hz), 7.15 (d, 1H, J 15.2 Hz), 6.91 (d, 1H, J 15.2 Hz), 2.80 (ddd, 1H, J 12.0, 6.8, 6.0 Hz), 2.35-2.25 (m, 2H), 2.20 (s, 3H), 2.05-1.95 (m, 2H), 1.95-1.85 (m, 1H); ${ }^{13} \mathrm{C}$ NMR $\delta 212.36,200.54,188,65,144.79$, $140.22,135.92,130.47,129.51,126.87,72.20,38.58,32.06,27.84,19.91 ;[\alpha]^{\mathrm{rt}}=-18.2$ $\left(c=2.30 \mathrm{~g} / 100 \mathrm{~mL}, \mathrm{CHCl}_{3}, 80 \%\right.$ ee - reaction performed at room temperature).

(Z)-3d: ${ }^{1} \mathrm{H}$ NMR $\delta 7.81(\mathrm{~d}, 2 \mathrm{H}, J 8.8 \mathrm{~Hz}), 7.39$ (d, 2H, $\left.J 8.8 \mathrm{~Hz}\right), 7.19$ (d, 1H, $\left.J 12.0 \mathrm{~Hz}\right)$, $6.53(\mathrm{~d}, 1 \mathrm{H}, J 12.0 \mathrm{~Hz}), 2.95-2.85(\mathrm{~m}, 1 \mathrm{H}), 2.35-2.25(\mathrm{~m}, 2 \mathrm{H}), 2.15$ (s, 3H), 2.05-1.95 (m, 2H), 1.95-1.85 (m, 1H); ${ }^{13} \mathrm{C}$ NMR $\delta 214.03,200.47,189.99,145.61,140.40,135.82$, $130.35,129.52,126.69,72.19,37.98,33.76,27.69$, 20.14; HRMS calc.: $\mathrm{C}_{16} \mathrm{H}_{15} \mathrm{ClNaO}_{3}$ 313.0607; found: 313.0607 .

3e: The ee was determined by HPLC using Chiralpak AD column (hexane/ $i$-PrOH (80:20); flow rate $1.0 \mathrm{ml} / \mathrm{min} ;(Z)-3 \mathbf{e}: \tau_{\text {minor }}=16.9 \mathrm{~min}, \tau_{\text {major }}=18.3 \mathrm{~min} ;(E)-3 \mathbf{e}: \tau_{\text {minor }}=$ $\left.20.0 \mathrm{~min}, \tau_{\text {major }}=24.7 \mathrm{~min}\right)$.

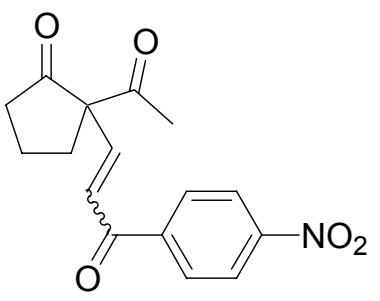

Yield: 99\%

(E)-3e: ${ }^{1} \mathrm{H}$ NMR $\delta 8.24(\mathrm{~d}, 2 \mathrm{H}, J 8.8 \mathrm{~Hz}), 7.98(\mathrm{~d}, 2 \mathrm{H}, J 8.8 \mathrm{~Hz}), 7.19(\mathrm{~d}, 1 \mathrm{H}, J 15.8 \mathrm{~Hz})$, $6.92(\mathrm{~d}, 1 \mathrm{H}, J 15.8 \mathrm{~Hz}), 2.82$ (ddd, 1H, J 12.0, 6.8, 6.0 Hz), 2.20-2.10 (m, 2H), 2.13 (s, $3 \mathrm{H}), 2.05-1.95(\mathrm{~m}, 2 \mathrm{H}), 1.90-1.80(\mathrm{~m}, 1 \mathrm{H})$. 
(Z)-3e: ${ }^{1} \mathrm{H}$ NMR $\delta 8.25$ (d, 2H, $\left.J 8.8 \mathrm{~Hz}\right), 7.99$ (d, 2H, J $\left.8.8 \mathrm{~Hz}\right), 6.96$ (d, 1H, $J 12.0 \mathrm{~Hz}$ ), 6.86 (d, 1H, J 12.0 Hz), 2.90-2.80 (m, 1H), 2.30-2.20 (m, 2H), 2.19 (s, 3H), 2.05-1.95 (m, $2 \mathrm{H}), 1.95-1.85(\mathrm{~m}, 1 \mathrm{H}) ;{ }^{13} \mathrm{C}$ NMR (mixture of isomers) $\delta 213.58,212.13,200.32,200.10$, $189.58,188.52,150.79,146.29,142.29,141.96,130.01,129.93,126.66,126.38,124.53$, $72.32,72.13,38.49,37.95,33.87,32.02,27.80,27.68,20.08$; HRMS calc.: $\mathrm{C}_{16} \mathrm{H}_{15} \mathrm{NaNO}_{5}$ 324.0847; found: 324.0851 .

3f: The ee was determined by HPLC using Chiralpak AS column (hexane $/ i-\mathrm{PrOH}$ (80:20); flow rate $1.0 \mathrm{~mL} / \mathrm{min}$; $(E)-3 \mathbf{3 f :} \tau_{\text {minor }}=5.7 \mathrm{~min}, \tau_{\text {major }}=6.7 \mathrm{~min} ;(Z)-3 \mathbf{f}: \tau_{\operatorname{minor}}=$ $\left.13.9 \mathrm{~min}, \tau_{\text {major }}=34.4 \mathrm{~min}\right)$.

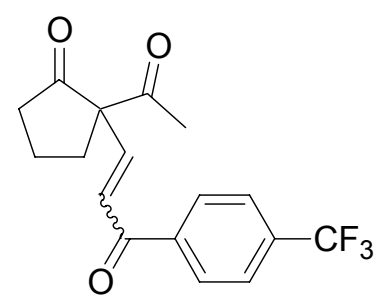

Yield: 99\%

(E)-3f: ${ }^{1} \mathrm{H}$ NMR $\delta 7.94$ (d, 2H, $\left.J 8.4 \mathrm{~Hz}\right), 7.68$ (d, 2H, $\left.J 8.4 \mathrm{~Hz}\right), 7.19$ (d, $\left.1 \mathrm{H}, J 15.8 \mathrm{~Hz}\right)$, 6.93 (d, 1H, $J 15.8 \mathrm{~Hz}$ ), 2.95 (ddd, 1H, $J$ 12.0, 6.8, $6.0 \mathrm{~Hz}$ ), 2.4-2.2 (m, 2H), 2.21 (s, 3H), 2.1-1.9 (m, 3H), 1.95-1.85 (m, 1H); ${ }^{13} \mathrm{C}$ NMR $\delta 212.27,200.34,189.16,145.63,140.05$, 134.93 (q, $J(F) 32.8 \mathrm{~Hz}), 129.38,126.95,126.25$ (q, $J(F) 3.8 \mathrm{~Hz}), 125.92$ (q, $J(F) 122.0$ $\mathrm{Hz}), 72.32,38.57,32.06,27.54,20.08 ;{ }^{19} \mathrm{~F}$ NMR $\delta-63.6 ;[\alpha]^{\mathrm{rt}}{ }_{\mathrm{D}}=-10.8(c=2.90 \mathrm{~g} / 100$ $\mathrm{mL}, \mathrm{CHCl}_{3}, 85 \%$ ee - reaction performed at room temperature).

(Z)-3f: ${ }^{1} \mathrm{H}$ NMR $\delta 7.96$ (d, 2H, $\left.J 8.8 \mathrm{~Hz}\right), 7.68$ (d, 2H, $\left.J 8.8 \mathrm{~Hz}\right), 6.96(\mathrm{~d}, 1 \mathrm{H}, J 11.6 \mathrm{~Hz})$, 6.67 (d, 1H, J 11.6 Hz), 3.0-2.9 (m, 1H), 2.35-2.25 (m, 2H), 2.13 (s, 3H), 2.0-1.9 (m, $3 \mathrm{H}) ;{ }^{13} \mathrm{C}$ NMR $\delta 213.81,200.44,190.26,146.12,139.05,134.73(\mathrm{q}, J(F) 32.8 \mathrm{~Hz}$ ), 129.26, 126.95, 126.24 (q, $J(F) 3.8 \mathrm{~Hz}), 125.92$ (q, $J(F) 122.0 \mathrm{~Hz}$ ), 72.17, 39.19, 33.72, 27.77, 19.88; ${ }^{19} \mathrm{~F}$ NMR $\delta$-63.6. HRMS calc.: $\mathrm{C}_{17} \mathrm{H}_{15} \mathrm{~F}_{3} \mathrm{NaO}_{3}$ 347.0871; found: 347.0874 . 
3g: The ee was determined by HPLC using Chiralpak AD column (hexane $/ i-\mathrm{PrOH}$ (85:15); flow rate $1.0 \mathrm{~mL} / \mathrm{min} ;(Z)-3 \mathbf{3 g}: \tau_{\text {minor }}=12.8 \mathrm{~min}, \tau_{\text {major }}=14.0 \mathrm{~min} ;(E)-3 \mathbf{g}: \tau_{\text {minor }}=$ $17.6 \mathrm{~min}, \tau_{\text {major }}=21.3 \mathrm{~min}$ ).

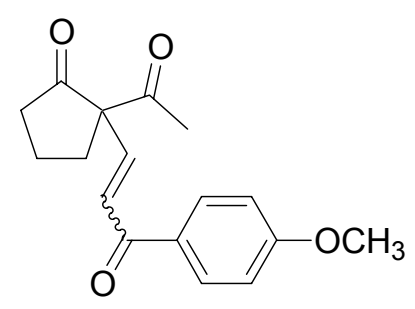

Yield: 99\%

(E)-3g: ${ }^{1} \mathrm{H}$ NMR $\delta 7.86$ (d, 2H, $\left.J 8.8 \mathrm{~Hz}\right), 7.10$ (d, $\left.1 \mathrm{H}, J 15.8 \mathrm{~Hz}\right), 6.88$ (d, 2H, $\left.J 8.8 \mathrm{~Hz}\right)$, 6.86 (d, 1H, $J 15.8 \mathrm{~Hz}), 3.87$ (s, 3H), 2.82 (ddd, 1H, $J 12.0,6.8,6.0 \mathrm{~Hz}), 2.30-2.20$ (m, $2 \mathrm{H}), 2.13(\mathrm{~s}, 3 \mathrm{H}), 2.0-1.4(\mathrm{~m}, 3 \mathrm{H})$.

(Z)-3g: ${ }^{1} \mathrm{H}$ NMR $\delta 7.85$ (d, 2H, J 8.8 Hz), 6.96 (d, $\left.1 \mathrm{H}, J 12.0 \mathrm{~Hz}\right), 6.90$ (d, 2H, $J 8.8 \mathrm{~Hz}$ ), $6.55(\mathrm{~d}, 1 \mathrm{H}, J 12.0 \mathrm{~Hz}), 3.86(\mathrm{~s}, 3 \mathrm{H}), 2.95-2.85(\mathrm{~m}, 1 \mathrm{H}), 2.3-2.2(\mathrm{~m}, 1 \mathrm{H}), 2.20(\mathrm{~s}, 3 \mathrm{H})$, 2.0-1.4 (m, 4H); ${ }^{13} \mathrm{C}$ NMR (mixture of isomers) $\delta 214.43,212.58,201.01,200.73$, $189.73,188.61,164.24,164.18,144.13,143.30,131.43,131.29,130.59,130.54,127.27$, 127.21, 114.36 (2 peaks), 72.10, 72.05, 55.97, 55.89, 38.60, 37.99, 33.75, 32.09, 27.84, 27.68, 20.15, 19.87; HRMS calc.: $\mathrm{C}_{17} \mathrm{H}_{18} \mathrm{NaO}_{4}$ 309.1102; found: 309.1100 .

3h: The ee was determined by HPLC using Chiralpak AS column (hexane $/ i-\mathrm{PrOH}$ (90:10); flow rate $1.0 \mathrm{~mL} / \mathrm{min}$; $(E)-3 \mathbf{h}: \tau_{\text {major }}=11.8 \mathrm{~min}, \tau_{\operatorname{minor}}=12.6 \mathrm{~min}$; hexane $/ i$ PrOH (65:35); flow rate $1.0 \mathrm{~mL} / \mathrm{min}$; $\left.(Z)-3 \mathbf{h}: \tau_{\text {major }}=9.8 \mathrm{~min}, \tau_{\text {minor }}=11.6 \mathrm{~min}\right)$.

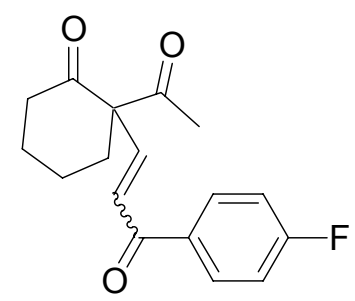

Yield: 95\%

(E)-3h: ${ }^{1} \mathrm{H}$ NMR $\delta$ 7.9-7.8 (m, 2H), 7.32 (d, 1H, $\left.J 16 \mathrm{~Hz}\right), 7.1-7.0$ (m, 2H), $6.70(\mathrm{~d}, 1 \mathrm{H}, J$ $16 \mathrm{~Hz}$ ), 2.2-2.6 (m, 4H), 2.08 (s, 3H), 2.0-1.7 (m, 4H). 
(Z)-3h: ${ }^{1} \mathrm{H}$ NMR $\delta$ 7.9-7.8 (m, 2H), 7.1-7.0 (m, 2H), 6.95 (d, 1H, $\left.J 12 \mathrm{~Hz}\right), 6.55$ (d, 1H, $J$ $12.4 \mathrm{~Hz}$ ), 2.2-2.6 (m, 4H), $2.05(\mathrm{~s}, 3 \mathrm{H}), 2.0-1.7(\mathrm{~m}, 4 \mathrm{H}) ;{ }^{13} \mathrm{C}$ NMR (mixture of isomers) $\delta$ 207.18, (2 peaks) 203.24, 202.57, 189.75, 188.69, 166.16 (2 peaks) (d, $J(F) 257.3 \mathrm{~Hz}$ ), 145.10, 144.61, 134.01 (d, $J(F) 3 \mathrm{~Hz}$ ), 133.98 (d, $J(F) 3.0 \mathrm{~Hz}$ ), 132.89, 132.79, 131.75, (d, $J(F) 9.8 \mathrm{~Hz}), 131.65,(\mathrm{~d}, J(F) 9.8 \mathrm{~Hz}), 127.68,126.94,70.68,70.33,41.80,40.87$, $36.15,34.97,27.87,27.76,27.08,27.05,22.42,22.35 ;{ }^{19} \mathrm{~F}$ NMR $\delta-104.8,(\mathrm{~m}),-105.1$ (m); HRMS calc.: $\mathrm{C}_{17} \mathrm{H}_{17} \mathrm{FNaO}_{3} 311.1059$; found: 311.1062 .

3i: The ee was determined by HPLC using Chiralpak AS column (hexane $/ i-\mathrm{PrOH}$ (90:10); flow rate $1.0 \mathrm{~mL} / \mathrm{min} ;(E)-3 \mathbf{i}: \tau_{\text {major }}=13.0 \mathrm{~min}, \tau_{\text {minor }}=15.1 \mathrm{~min}$; hexane $/ \mathrm{i}-\mathrm{PrOH}$ (50:50); flow rate $1.0 \mathrm{~mL} / \mathrm{min}$; $(Z)-3 \mathrm{i}: \tau_{\text {major }}=10.2 \mathrm{~min}, \tau_{\text {minor }}=14.5 \mathrm{~min}$ ).

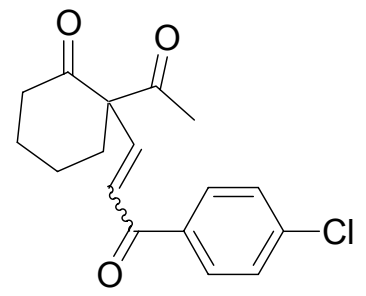

Yield: $95 \%$

(E)-3i: ${ }^{1} \mathrm{H}$ NMR $\delta 7.81$ (d, 2H, $\left.J 8.8 \mathrm{~Hz}\right), 7.40$ (d, 2H, $\left.J 8.8 \mathrm{~Hz}\right), 7.37$ (d, 1H, $\left.J 16.4 \mathrm{~Hz}\right)$, $6.73(\mathrm{~d}, 1 \mathrm{H}, J 16.4 \mathrm{~Hz}), 2.2-2.6(\mathrm{~m}, 4 \mathrm{H}), 2.12(\mathrm{~s}, 3 \mathrm{H}), 2.0-1.7(\mathrm{~m}, 4 \mathrm{H}) ;[\alpha]_{\mathrm{D}}^{\mathrm{rt}}=+61.9(c=$ $0.85 \mathrm{~g} / 100 \mathrm{~mL}, \mathrm{CHCl}_{3}, 85 \%$ ee - reaction performed at room temperature).

(Z)-3i: ${ }^{1} \mathrm{H}$ NMR $\delta 7.85$ (d, 2H, $\left.J 8.8 \mathrm{~Hz}\right), 7.40$ (d, 2H, $\left.J 8.8 \mathrm{~Hz}\right), 6.99$ (d, 1H, $J 12.4 \mathrm{~Hz}$ ), $6.62(\mathrm{~d}, 1 \mathrm{H}, J 12.4 \mathrm{~Hz}), 2.2-2.6(\mathrm{~m}, 4 \mathrm{H}), 2.10(\mathrm{~s}, 3 \mathrm{H}), 2.0-1.7(\mathrm{~m}, 4 \mathrm{H}) ;{ }^{13} \mathrm{C}$ NMR (mixture of isomers) $\delta 207.09,206.84,203.17,203.16,190.04,189.03,145.42,144.87$, $140.25,140.02,135.93,135.85,130.52,130.38,129.42,129.40,127.55,126.84,70.65$ (2 peaks), 41.78, 40.87, 36.13, 34.93, 27.84, 27.75, 27.03, 26.97, 22.40, 22.33; HRMS calc.: $\mathrm{C}_{17} \mathrm{H}_{17} \mathrm{ClNaO}_{3}$ 341.1154; found: 341.1151 .

3j: The ee was determined by HPLC using Chiralpak AD column (hexane $/ i-\mathrm{PrOH}$ (85:15); flow rate $1.0 \mathrm{~mL} / \mathrm{min} ;(Z)-\mathbf{3 j}: \tau_{\text {major }}=16.1 \mathrm{~min}, \tau_{\text {minor }}=17.6 \mathrm{~min} ;(E)-\mathbf{3} \mathbf{j}: \tau_{\text {major }}$ $=21.9 \mathrm{~min}, \tau_{\text {minor }}=25.9 \mathrm{~min}$ ). 


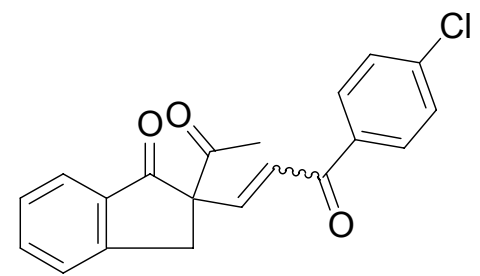

Yield: 95\%

${ }^{1} \mathrm{H}$ NMR (mixture of isomers) $\delta$ 7.6-7.0 (m, $\left.20 \mathrm{H}\right), 4.15(\mathrm{~d}, 1 \mathrm{H}, J 17.2 \mathrm{~Hz}), 4.05(\mathrm{~d}, 1 \mathrm{H}, J$ $17.2 \mathrm{~Hz}), 3.13$ (d, 1H, J 17.2 Hz), 2.70 (d, 1H, J 17.2 Hz), 2.35 (s, 3H), $2.25(\mathrm{~s}, 3 \mathrm{H}) ;{ }^{13} \mathrm{C}$ NMR (mixture of isomers) $\delta 201.58,198.90,199.61,194.48,189.67,188.37,154.82$, $153.01,146.38,145.05,140.50,140.09,136.46,136.16,135.90,135.76,134.30,134.00$, $130.45,130.32$, 129.53, 129.41, 128.52, 128.09, 126.82, 126.71, 126.59, 126.52, 125.43, 125.18, 72.83, 72.27, 38.55, 35.73, 27.50, 27.28. HRMS calc.: $\mathrm{C}_{20} \mathrm{H}_{15} \mathrm{ClNaO}_{3}$ 361.0607; found: 361.0570 .

3k: The ee was determined by HPLC using Chiralpak AD column (hexane $/ i$-PrOH (90:10); flow rate $1.0 \mathrm{~mL} / \mathrm{min} ;(Z)-3 \mathbf{k}: \tau_{\text {major }}=17.7 \mathrm{~min}, \tau_{\text {minor }}=19.2 \mathrm{~min} ;(E)-3 \mathbf{k}: \tau_{\text {major }}$ $=22.4 \mathrm{~min}, \tau_{\text {minor }}=23.0 \mathrm{~min}$ ).

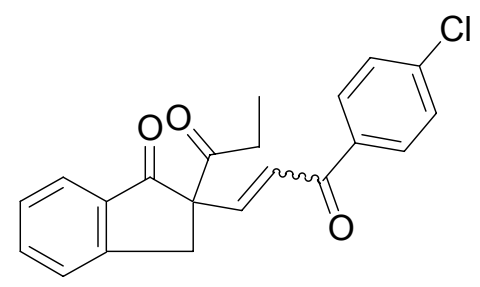

Yield: 95\%

${ }^{1} \mathrm{H}$ NMR (mixture of isomers) $\delta$ 7.6-7.0 (m, $\left.20 \mathrm{H}\right), 4.19$ (d, 1H, $\left.J 17.4 \mathrm{~Hz}\right), 4.04(\mathrm{~d}, 1 \mathrm{H}, J$ $17.4 \mathrm{~Hz}), 3.15$ (d, 1H, J 17.4 Hz), 2.70 (d, 1H, J 17.4 Hz), 2.8-2.5 (m, 4H), 0.94 (t, 3H, J $6.8 \mathrm{~Hz}$ ), 0.92 (t, 3H, $J 6.8 \mathrm{~Hz}$ ); ${ }^{13} \mathrm{C}$ NMR (mixture of isomers) $\delta 202.95,201.81,201.48$, $199.91,189.75,188.91,155.05,153.20,146.76,145.41,140.52,140.18,136.48,136.20$, 136.02 , 135.87, 134.46, 134.09, 130.53, 130.39, 129.61, 129.49, 128.68, 128.55, 126.85, $126.59,126.53,126.49,125.75,125.52,72.86,71.99,38.98,36.27,33.61,32.90,8.39$, 8.37; HRMS calc.: $\mathrm{C}_{21} \mathrm{H}_{17} \mathrm{ClNaO}_{3}$ 375.0764; found: 375.0762 . 
31: The ee was determined by HPLC using 2 Chiralpak AD columns (hexane $/ i-\mathrm{PrOH}$ (95:5); flow rate $1.0 \mathrm{~mL} / \mathrm{min}$; $(Z)-31: \tau_{\text {major }}=48.7 \mathrm{~min}, \tau_{\text {minor }}=51.7 \mathrm{~min} ;(E)-31: \tau_{\text {minor }}=$ $\left.57.1 \mathrm{~min}, \tau_{\text {major }}=60.1 \mathrm{~min}\right)$.

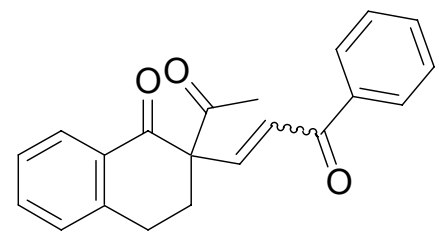

Yield: 99\%

(E)-3I: ${ }^{1} \mathrm{H}$ NMR $\delta 8.06(\mathrm{~d}, 1 \mathrm{H}, J 8.0 \mathrm{~Hz}), 7.82(\mathrm{~d}, 2 \mathrm{H}, J 8.0 \mathrm{~Hz}), 7.6-7.0(\mathrm{~m}, 7 \mathrm{H}), 6.84(\mathrm{~d}$, $1 \mathrm{H}, J 15.6 \mathrm{~Hz}$ ), 3.3-2.6 (m, 4H) 2.18 (s, 3H).

(Z)-3I: ${ }^{1} \mathrm{H}$ NMR $\delta 7.92(\mathrm{~d}, 1 \mathrm{H}, J 8.0 \mathrm{~Hz}), 7.87$ (d, 2H, $\left.J 8.0 \mathrm{~Hz}\right), 7.6-7.0(\mathrm{~m}, 6 \mathrm{H}), 7.04(\mathrm{~d}$, $1 \mathrm{H}, J 12.0 \mathrm{~Hz}), 6.51(\mathrm{~d}, 1 \mathrm{H}, J 12.0 \mathrm{~Hz}), 3.3-2.6(\mathrm{~m}, 4 \mathrm{H}) 2.18(\mathrm{~s}, 3 \mathrm{H}) ;{ }^{13} \mathrm{C}$ NMR (mixture of isomers) $\delta 203.81,202.96,196.69,195.16,191.31,190.20,144.84,143.96,143.73$, $137.63,137.59,134.83,134.33,133.82,133.61,132.30,131.97,131.84,129.37,129.23$, $129.11,129.09,129.00,128.64,128.51,128.35,128.09,127.99,127.52,127.25,127.19$, 126.21, 66.98, 66.77, 31.13, 30.93, 28.87, 28.73, 26.64, 26.10; HRMS calc.: $\mathrm{C}_{21} \mathrm{H}_{18} \mathrm{NaO}_{3}$ 341.1154; found: 341.1154 .

3m: The ee was determined by HPLC using Chiralpak AD column (hexane $/ i$-PrOH (85:15); flow rate $1.0 \mathrm{~mL} / \mathrm{min} ;(Z)-3 \mathbf{m} \tau_{\text {major }}=15.7 \mathrm{~min}, \tau_{\text {minor }}=18.0 \mathrm{~min} ;(E)-\mathbf{3 m} ; \tau_{\text {minor }}$ $=20.3 \mathrm{~min}, \tau_{\text {major }}=22.9 \mathrm{~min}$ ).

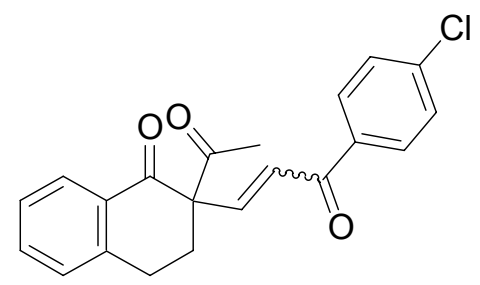

Yield: 99\%

(E)-3m: ${ }^{1} \mathrm{H}$ NMR $\delta 8.04$ (d, 1H, J 8.0 Hz), 7.92 (d, 1H, J 8.0 Hz), 7.81 (d, 2H, J $8.8 \mathrm{~Hz}$ ), 7.75 (d, 2H, J 8.8 Hz), 7.35 (d, 4H, J 8.8 Hz), 7.4-7.0 (m, 7H), $6.97(Z, \mathrm{~d}, 1 \mathrm{H}, J 12.0 \mathrm{~Hz})$, $6.80(E, \mathrm{~d}, 1 \mathrm{H}, J 16.0 \mathrm{~Hz}), 6.48(\mathrm{Z}, \mathrm{d}, 1 \mathrm{H}, J 12.0 \mathrm{~Hz}), 3.3-3.2(\mathrm{~m}, 1 \mathrm{H}), 3.1-3.0(\mathrm{~m}, 1 \mathrm{H})$, 
2.9-2.4 (m, 4H), 2.4-2.3 (m, 1H), 2.2-2.1 (m, 1H), $2.17(\mathrm{~s}, 6 \mathrm{H}) ;[\alpha]^{\mathrm{rt}}=-1.0(c=3.55 \mathrm{~g} /$ $100 \mathrm{~mL}, \mathrm{CHCl}_{3}, 84 \%$ ee - reaction performed at room temperature).

(Z)-3m: ${ }^{1} \mathrm{H}$ NMR $\delta 8.25(\mathrm{~d}, 2 \mathrm{H}, J 8.8 \mathrm{~Hz}), 7.99(\mathrm{~d}, 2 \mathrm{H}, J 8.8 \mathrm{~Hz}), 6.96(\mathrm{~d}, 1 \mathrm{H}, J 12.0 \mathrm{~Hz})$, 6.68 (d, $1 \mathrm{H}, J 12.0 \mathrm{~Hz}), 2.90-2.80$ (m, 1H), 2.3-2.2 (m, 2H), 2.19 (s, 3H), 2.05-1.95 (m, $2 \mathrm{H}), 1.95-1.85(\mathrm{~m}, 1 \mathrm{H}) ;{ }^{13} \mathrm{C}$ NMR (mixture of isomers) $\delta 203.80,202.77,195.36,195.7$, $190.17,188.91,145.37,144.02,143.80,143.72,140.23,140.03,135.93$ (2 peaks), $134.89,134.35,132.02,131.78,130.52,130.42,129.41,129.40,129.39,128.61,128.52$, $127.85,127.80,127.55,127.24,67.00,66.78,31.47,30.97,28.82,28.66,26.59,26.08$. 33.87, 32.02, 27.80, 27.68, 20.08, 19.91; HRMS calc.: $\mathrm{C}_{21} \mathrm{H}_{17} \mathrm{ClNaO}_{3}$ 375.0764; found: 375.0767 .

3n: The ee was determined by HPLC using Chiralcel OJ column (hexane/i-PrOH (80:20); flow rate $1.0 \mathrm{~mL} / \mathrm{min} ;(E)-\mathbf{3 n}: \tau_{\text {major }}=21.3 \mathrm{~min} ; \tau_{\text {minor }}=30.0 \mathrm{~min} ;(Z)-\mathbf{3 n}: \tau_{\text {major }}=34.1$ $\left.\min , \tau_{\text {minor }}=43.0 \mathrm{~min}\right)$.

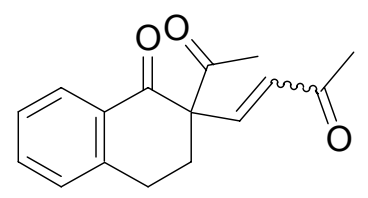

Yield: 97\% (E)-3n: ${ }^{1} \mathrm{H}$ NMR $\delta 8.01(\mathrm{~d}, 1 \mathrm{H}, J 8.0 \mathrm{~Hz}), 7.5-7.1(\mathrm{~m}, 4 \mathrm{H}), 7.23(\mathrm{~d}, 1 \mathrm{H}, J$ $16.4 \mathrm{~Hz}), 6.02$ (d, 1H, J 16.4 Hz), 3.2-2.5 (m, 4H); 2.23 (s, 3H), 2.21 (s, 3H).

(Z)-3n: ${ }^{1} \mathrm{H}$ NMR $\delta 7.95(\mathrm{~d}, 1 \mathrm{H}, J 8.0 \mathrm{~Hz}), 7.5-7.1(\mathrm{~m}, 4 \mathrm{H}), 6.37$ (d, $\left.1 \mathrm{H}, J 11.6 \mathrm{~Hz}\right), 6.15$ (d, $1 \mathrm{H}, J 11.6 \mathrm{~Hz}$ ), 3.2-2.5 (m, 4H); $2.16(\mathrm{~s}, 3 \mathrm{H}), 2.11(\mathrm{~s}, 3 \mathrm{H}) ;{ }^{13} \mathrm{C}$ NMR (mixture of isomers) $\delta 204.03,202.35,198.62,198.08,195.22,195.07,143.86,143.74,143.59$, $142.58,134.90,134.45,134.22,132.84,132.28,131.78,130.25,129.37,129.27,128.53$, $127.53,127.30,66.69,66.64,31.64,31.56,30.56,28.79,28.28,27.75,26.67,26.04$.

3o: The ee was determined by HPLC using Chiralcel OJ column (hexane/i-PrOH (80:20); flow rate $1.0 \mathrm{~mL} / \mathrm{min} ;(E)$-3o: $\tau_{\text {major }}=29.7 \mathrm{~min} ; \tau_{\text {minor }}=35.2 \mathrm{~min} ;(Z)-30: \tau_{\text {major }}=42.5$ $\left.\min , \tau_{\text {minor }}=50.5 \mathrm{~min}\right)$. 


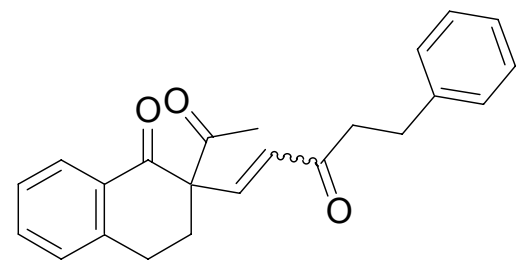

Yield: 97\% (E)-3o: ${ }^{1} \mathrm{H}$ NMR $\delta 7.99(\mathrm{~d}, 1 \mathrm{H}, J 8.0 \mathrm{~Hz}), 7.5-7.1(\mathrm{~m}, 10 \mathrm{H}), 6.02(\mathrm{~d}, 1 \mathrm{H}, J$ $16.4 \mathrm{~Hz}), 2.8-2.2(\mathrm{~m}, 8 \mathrm{H}), 2.07(\mathrm{~s}, 3 \mathrm{H})$.

(Z)-3o: ${ }^{1} \mathrm{H}$ NMR $\delta 7.94$ (d, 1H, J 8.0 Hz), 7.5-7.1 (m, 9H), 6.32 (d, 1H, $\left.J 12.4 \mathrm{~Hz}\right), 6.17$ $(\mathrm{d}, 1 \mathrm{H}, J 12.4 \mathrm{~Hz}), 2.8-2.2(\mathrm{~m}, 8 \mathrm{H}), 2.07(\mathrm{~s}, 3 \mathrm{H}) ;{ }^{13} \mathrm{C}$ NMR (mixture of isomers) $\delta 203.90,202.48,199.97,199.06,195.42,195.06,143.78,143.75,143.12,142.86$, $141.26,141.23,134.89,134.29,132.31,132.13,132.05,131.78,129.95,129.37,128.94$, $128.91,128.80,128.75,128.60,128.54,127.53,127.28,126.60,126.57,66.95,66.62$, $47.34,45.80,42.50,31.40,30.57,30.31,29.97,28.40,26.68,26.04$. 


\section{Additional data on several combinations of alkynones/ $\beta$-dicarbonyl compounds.}

(Table 3)

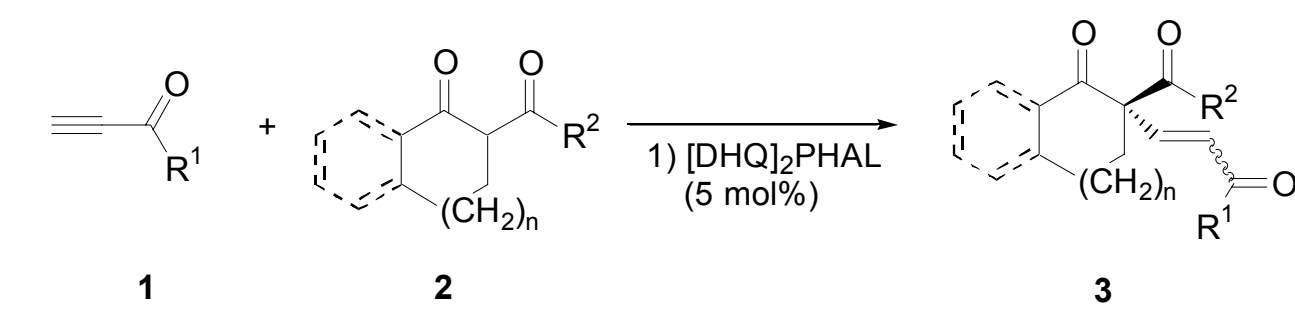

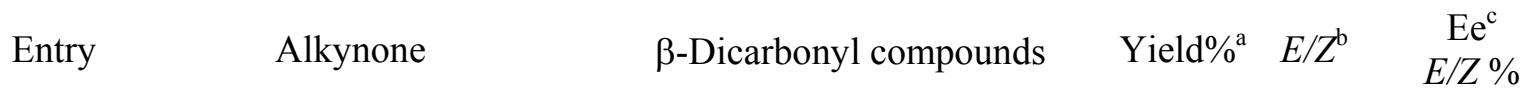

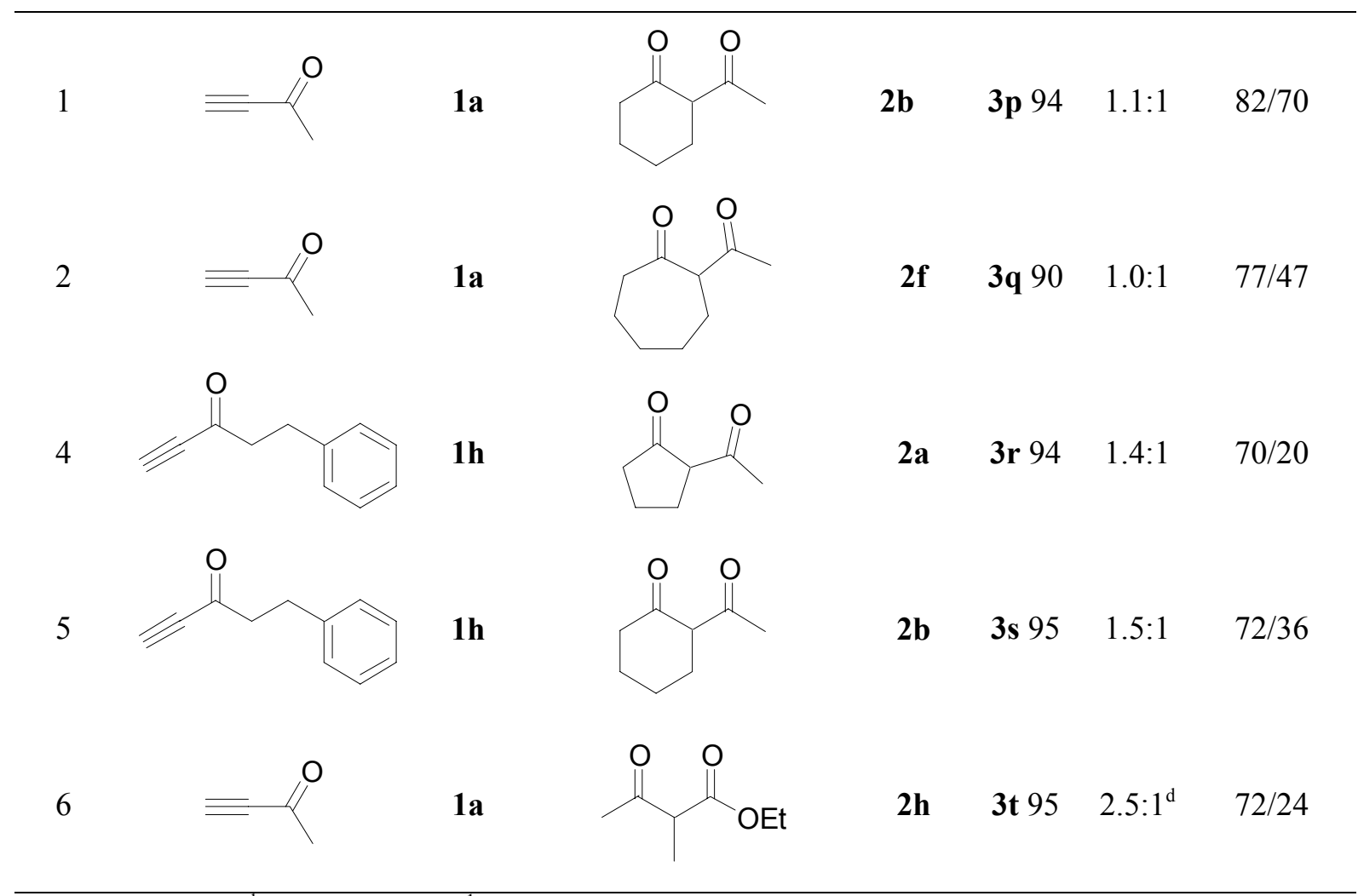

\footnotetext{
${ }^{a}$ Isolated yield. ${ }^{b}$ Determined by ${ }^{1}$ H-NMR. ${ }^{c}$ Enantiomeric excess determined by HPLC or CSP-GC. ${ }^{\mathrm{d}}$ Determined by GC.
}

3p: The ee was determined by HPLC using Chiralcel OJ columns (hexane $/ i-\mathrm{PrOH}$ (80:20); flow rate $1.0 \mathrm{~mL} / \mathrm{min} ;(E)-3 \mathbf{p}: \tau_{\text {major }}=12.0 \mathrm{~min} ; \tau_{\text {minor }}=14.0$. min; $(Z)-3 \mathbf{p}: \tau_{\text {major }}$ $\left.=15.6 \mathrm{~min} ; \tau_{\text {minor }}=17.0 \mathrm{~min}\right)$. 


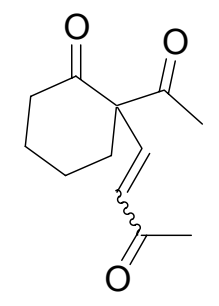

Yield :94\%

(E)-3p ${ }^{1} \mathrm{H}$ NMR $\delta 7.22$ (d, 1H, $\left.J 16.0 \mathrm{~Hz}\right), 5.94(\mathrm{~d}, 1 \mathrm{H}, J 16.0 \mathrm{~Hz}), 2.6-1.6(\mathrm{~m}, 8 \mathrm{H}), 2.25$ $(\mathrm{s}, 3 \mathrm{H}), 2.06(\mathrm{~s}, 3 \mathrm{H})$.

(Z)-3p: ${ }^{1} \mathrm{H}$ NMR $\delta 6.35$ (d, 1H, $\left.J 12.0 \mathrm{~Hz}\right), 6.23$ (d, 1H, $\left.J 12.0 \mathrm{~Hz}\right), 2.6-1.6(\mathrm{~m}, 8 \mathrm{H}), 2.17$ $(\mathrm{s}, 3 \mathrm{H}), 2.03(\mathrm{~s}, 3 \mathrm{H}) ;{ }^{13} \mathrm{C} \mathrm{NMR}$ (mixture of isomers) $\delta 207.10,206.70,203.40,202.21$, 198.71, 198.27, 143.86, 143.43, 132.70, 129.45, 70.56, 69.94, 41.75, 40.86, 35.98, 34.67, 31.56 (2 peaks), 27.50, 27.42, 26.99, 26.70, 22.45, 22.29; HRMS calc.: $\mathrm{C}_{12} \mathrm{H}_{16} \mathrm{NaO}_{3}$ 231.0997; found: 231.0999 .

3q: The ee was determined by HPLC using Chiralpak AS+AD columns (hexane/ $i$-PrOH (95:5); flow rate $1.0 \mathrm{~mL} / \mathrm{min} ;(Z)-3 q: \tau_{\text {minor }}=20.9 \mathrm{~min}, \tau_{\text {major }}=32.9$. $\mathrm{min} ;(E)-\mathbf{3 q}: \tau_{\text {minor }}$ $=39.6 \mathrm{~min}, \tau_{\text {major }}=42.2 \mathrm{~min}$ ).

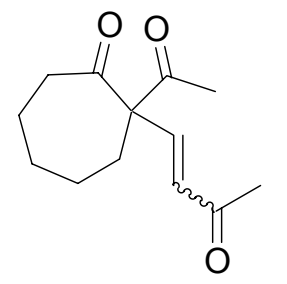

Yield: 94\%

(E)-3q ${ }^{1} \mathrm{H}$ NMR $\delta 7.28(\mathrm{~d}, 1 \mathrm{H}, J 16.4 \mathrm{~Hz}), 6.04(\mathrm{~d}, 1 \mathrm{H}, J 16.4 \mathrm{~Hz}), 2.8-1.2(\mathrm{~m}, 10 \mathrm{H}), 2.31$ $(\mathrm{s}, 3 \mathrm{H}), 2.11(\mathrm{~s}, 3 \mathrm{H})$.

(Z)-3q: ${ }^{1} \mathrm{H}$ NMR $\delta 6.78(\mathrm{~d}, 1 \mathrm{H}, J 12.0 \mathrm{~Hz}), 6.42(\mathrm{~d}, 1 \mathrm{H}, J 12.0 \mathrm{~Hz}), 2.6-1.6(\mathrm{~m}, 8 \mathrm{H}), 2.22$ $(\mathrm{s}, 3 \mathrm{H}), 1.94(\mathrm{~s}, 3 \mathrm{H}) ;{ }^{13} \mathrm{C} \mathrm{NMR}$ (mixture of isomers) $\delta 209.97,209.26,202.89,199.90$, $198.75,198.45,146.66,144.58,132.54,128.73,75.52,73.55,42.47,40.30,33.14,31.40$, $30.58,30.44,27.83,27.70,27.41,27.28,26.66,26.61,25.79,24.93$. 
3r: The ee was determined by HPLC using Chiralcel OD column (hexane $/ i-\mathrm{PrOH}$ (80:20); flow rate $1.0 \mathrm{~mL} / \mathrm{min} ;(Z)-3 \mathbf{r}: \tau_{\text {major }}=8.3 \mathrm{~min}, \tau_{\text {minor }}=9.1 \mathrm{~min} ;(E)-3 \mathbf{r}: \tau_{\text {major }}=$ $10.0 \mathrm{~min}, \tau_{\text {minor }}=11.5 \mathrm{~min}$ ).

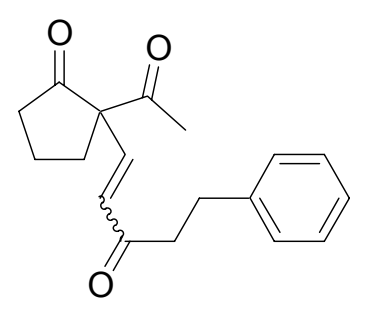

Yield: 94\%

(E)-3r: ${ }^{1} \mathrm{H}$ NMR $\delta$ 7.6-7.4 (m, 2H), 7.4-7.2 (m, 3H) 7.00 (d, 1H, J $\left.16.4 \mathrm{~Hz}\right), 6.13$ (d, 1H, $J 16.4 \mathrm{~Hz}), 3.0-2.7$ (m, 5H), 2.4-1.8 (m, 5H), 2.18, (s, 3H).

(Z)-3r: ${ }^{1} \mathrm{H}$ NMR $\delta$ 7.6-7.4 (m, 2H), 7.4-7.2 (m, 3H) $6.35(\mathrm{~d}, 1 \mathrm{H}, J 11.2 \mathrm{~Hz}), 6.30(\mathrm{~d}, 1 \mathrm{H}$, $J 11.2 \mathrm{~Hz}$ ), 3.0-2.7 (m, 5H), 2.4-1.8 (m, 5H), 2.10, (s, 3H); ${ }^{13} \mathrm{C}$ NMR (mixture of isomers) $\delta 214.00,212.40,200.66,200.39,199.83,199.00,143.78,142.46,141.17$, $141.10,131.38,129.05,128.94,128.89,128.79,128.74,126.62,126.58,72.01,71.89$, $45.69,42.64,38.37,37.99,33.82,31.54,30.28,29.92,27.61,27.44,20.13,19.79$.

3s: The ee was determined by HPLC using Chiralcel OJ columns (hexane $/ i-\mathrm{PrOH}$ (80:20); flow rate $1.0 \mathrm{~mL} / \mathrm{min} ;(Z)-3 \mathrm{~s}: \tau_{\text {major }}=24.3 \mathrm{~min}, \tau_{\text {major }}=61.2 \mathrm{~min} ;(E)-3 \mathrm{~s}: \tau_{\text {major }}=$ $\left.28.9 \mathrm{~min}, \tau_{\text {major }}=32.1 \mathrm{~min}\right)$.

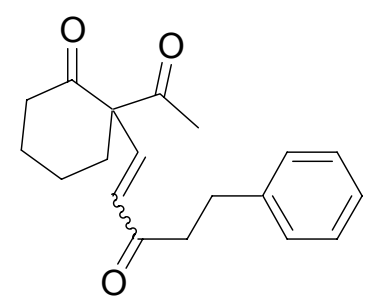

Yield: 95\%

(E)-3r: ${ }^{1} \mathrm{H}$ NMR $\delta$ 7.6-7.4 (m, 2H), 7.4-7.2 (m, 4H), 5.93 (d, 1H, $\left.J 16.0 \mathrm{~Hz}\right), 3.0-1.4(\mathrm{~m}$, 12H), 1.99 (s, 3H).

(Z)-3s: ${ }^{1} \mathrm{H}$ NMR $\delta$ 7.6-7.4 (m, 2H), 7.4-7.2 (m, 3H) $6.29(\mathrm{~d}, 1 \mathrm{H}, J 11.2 \mathrm{~Hz}), 6.25(\mathrm{~d}, 1 \mathrm{H}$, $J 11.2 \mathrm{~Hz}$ ), 3.0-1.4 (m, 12H), $1.98(\mathrm{~s}, 3 \mathrm{H}) ;{ }^{13} \mathrm{C}$ NMR (mixture of isomers) $\delta$ 207.11, 
206.86, 203.27, 202.24, 199.98, 199.24, 143.70, 143.15, 141.24, 141.17, 131.91, 129.09, $128.92,128.87,128.79,128.76,126.59,126.55,70.71,69.94,45.74,42.19,41.71,40.77$, $36.03,34.67,30.35,30.10,29.94,27.51,26.99,26.86,22.40,22.31$; HRMS calc. $\mathrm{C}_{19} \mathrm{H}_{22} \mathrm{NaO}_{3}$ : 321.1467; found: 321.1463 .

3t: The ee was determined by CSP-GC using a Chrompack CP-Chirasil Dex C $\beta$ column : rate $10{ }^{\circ} \mathrm{C} / \mathrm{min}$, starting $\mathrm{T}=70{ }^{\circ} \mathrm{C}$, $\mathrm{T}$ fin $200{ }^{\circ} \mathrm{C}$ for $10 \mathrm{~min}$; $(Z)-3 \mathrm{t}$ : $\tau_{\text {major }}=9.4 \mathrm{~min}, \tau$ minor $=9.5 \mathrm{~min} ;(E)-3 \mathrm{t}: \tau_{\text {minor }}=9.8 \mathrm{~min}, \tau_{\text {major }}=9.9 \mathrm{~min}$.

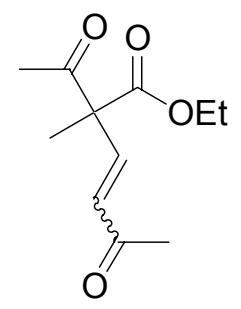

Yield: 94\%

(E)-3t: ${ }^{1} \mathrm{H}$ NMR $\delta 7.16$ (d, 1H, $\left.J 16.4 \mathrm{~Hz}\right), 6.02(\mathrm{~d}, 1 \mathrm{H}, J 16.0 \mathrm{~Hz}), 4.19$ (dq, $1 \mathrm{H}, J$ 7.2, $2.0 \mathrm{~Hz}), 2.25$ (s, 3H), 2.13 (s, 3H), 1.47 (s, 3H) 1.22 (t, 3H, J 7.2 Hz).

(Z)-3t: ${ }^{1} \mathrm{H}$ NMR $\delta 6.61$ (d, 1H, $\left.J 11.6 \mathrm{~Hz}\right), 6.24$ (d, 1H, $\left.J 11.6 \mathrm{~Hz}\right), 4.10$ (q, 1H, J 7.2, $\mathrm{Hz}$ ), $2.21(\mathrm{~s}, 3 \mathrm{H}), 2.14(\mathrm{~s}, 3 \mathrm{H}), 1.51(\mathrm{~s}, 3 \mathrm{H}) 1.17(\mathrm{t}, 3 \mathrm{H}, J 7.2 \mathrm{~Hz}) ;{ }^{13} \mathrm{C}$ NMR (mixture of isomers) $\delta 202.86,202.02,198.66,198.20,171.44,170.80,144.24,144.04,131.95$, $127.63,62.68,62.33,62.23,62.01,31.59,27.74,27.08,27.03,21.30,19.70,14.39,14.36$.

\section{Determination of absolute configuration}

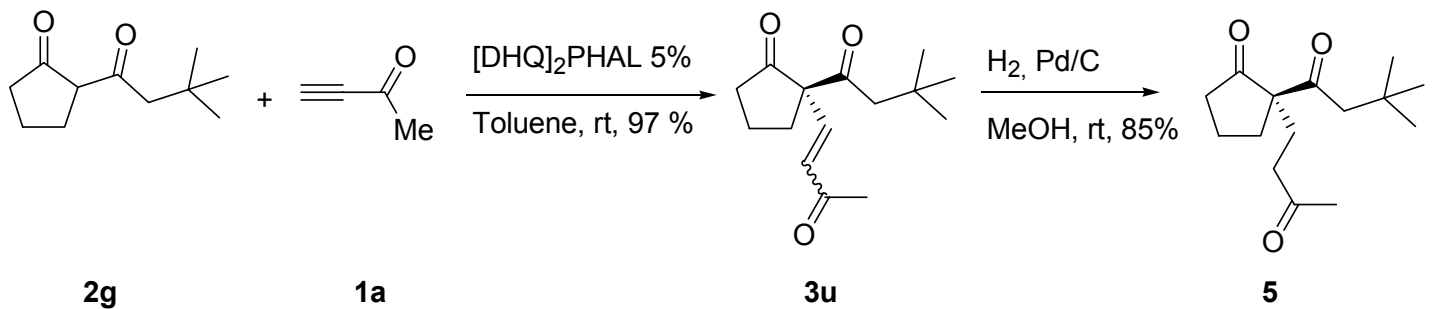

Absolute configuration was determined with comparison with the known compound 5 and extended by analogy to the other compounds. Compound $3 \mathbf{u}$ was prepared according to general procedure. Hydrogenation $\left(\mathrm{H}_{2}, \mathrm{Pd} / \mathrm{C} 10 \%, \mathrm{MeOH}, 85 \%\right.$ yield) gave product 5 found to be identical to the one reported (see below). ${ }^{5}$ 
3u: The ee was determined by HPLC using Chiralcel OJ column (hexane/i-PrOH (95:5); flow rate $1.0 \mathrm{~mL} / \mathrm{min} ;(Z)-3 \mathbf{u}: \tau_{\text {major }}=7.2 \mathrm{~min} ; \tau_{\text {minor }}=7.8 \mathrm{~min} ;(E)-\mathbf{3 u}: \tau_{\text {major }}=9.3 \mathrm{~min}$; $\left.\tau_{\text {minor }}=10.2 \mathrm{~min}\right) . E / Z$ ratio $(\mathrm{NMR})=1: 2: 1 ;(Z)-3 \mathbf{u}=26 \%$ ee; $(E)-3 \mathbf{u}=56 \%$ ee.

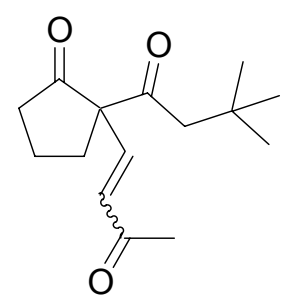

Yield: 97\%

(E)-3u: ${ }^{1} \mathrm{H}$ NMR $\delta 6.94$ (d, 1H, $\left.J 16.4 \mathrm{~Hz}\right), 6.02$ (d, 1H, $\left.J 16.4 \mathrm{~Hz}\right), 2.8-2.7$ (m, 1H), 2.4$2.2(\mathrm{~m}, 4 \mathrm{H}), 2.21,(\mathrm{~s}, 3 \mathrm{H}), 2.30-2.20(\mathrm{~m}, 2 \mathrm{H}, 2.0-1.8(\mathrm{~m}, 3 \mathrm{H}), 0.88(\mathrm{~s}, 9 \mathrm{H})$.

(Z)-3u: ${ }^{1} \mathrm{H}$ NMR $\delta 6.25$ (d, 1H, $\left.J 11.6 \mathrm{~Hz}\right), 6.21$ (d, 1H, $\left.J 11.6 \mathrm{~Hz}\right), 2.9-2.8(\mathrm{~m}, 1 \mathrm{H}), 2.4-$ $2.2(\mathrm{~m}, 4 \mathrm{H}), 2.13,(\mathrm{~s}, 3 \mathrm{H}), 2.30-2.20(\mathrm{~m}, 2 \mathrm{H}), 2.0-1.8(\mathrm{~m}, 3 \mathrm{H}), 0.87(\mathrm{~s}, 9 \mathrm{H}) ;{ }^{13} \mathrm{C} \mathrm{NMR}$ (mixture of isomers) $\delta 213.72,212.67,210.55,201.26,198.30,197.97,143.49,143.32$, 132.03, 129.09, 72.73, 72.64, 51.47, 51.40, 38.26, 37.88, 33.77, 31.58, 31.42, 31.18, $30.74,29.98,29.80,29.72,29.62,27.77,19.92,19.67$; HRMS calc.: $\mathrm{C}_{15} \mathrm{H}_{22} \mathrm{NaO}_{3}$ 273.1466 found: 273.1469 .

5: The ee was determined by HPLC using Chiralcel OJ column (hexane/i-PrOH (95:5); flow rate $0.5 \mathrm{~mL} / \mathrm{min} ; \tau_{\text {major }}=17.7 \mathrm{~min} ; \tau_{\text {minor }}=22.0 \mathrm{~min} ; 42 \%$ ee. $[\alpha]^{\mathrm{rt}}=+46.0(c=$ $4.78 \mathrm{~g} / 100 \mathrm{~mL}, \mathrm{CHCl}_{3}, 42 \%$ ee - reaction performed at room temperature).

Concordance of sign and magnitude of optical rotation, and HPLC retention times allowed us to assign $(R)$ as the absolute configuration of the product $\mathbf{5}$. Literature: Chiralcel OJ column (hexane $/ i-\mathrm{PrOH}$ (95:5); flow rate $0.44 \mathrm{~mL} / \mathrm{min} ; \tau_{\text {major }}=20.7 \mathrm{~min} ; \tau$ minor $=26.0 \mathrm{~min}$; ee $96 \%$. $[\alpha]_{\mathrm{D}}^{\mathrm{rt}}=+115.4\left(c=0.2 \mathrm{~g} / 100 \mathrm{~mL}, \mathrm{CHCl}_{3}, 96 \%\right.$ ee $){ }^{5}$ 


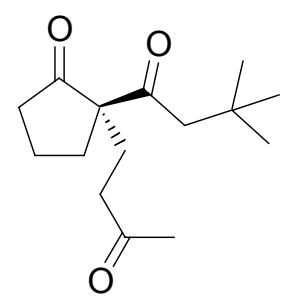

${ }^{1} \mathrm{H}$ NMR $\delta$ 2.04, (s, 3H), 2.4-1.6 (m, 11H), 1.6-1.4 (m, 1H) $0.90(\mathrm{~s}, 9 \mathrm{H}) ;{ }^{13} \mathrm{C}$ NMR $\delta$ 216.36, 207.20, 205.68, 67.93, 49.46, 38.45, 38.41, 31.58, 30.62, 29.88, 29.33, 27.20, 19.25; HRMS calc.: $\mathrm{C}_{15} \mathrm{H}_{24} \mathrm{NaO}_{3} 275.16231$ found: 275.1620 .

\footnotetext{
${ }^{1}$ Maeda, Y.; Kakiuchi, N.; Matsumura, S.; Nishimura, T.; Kawamura, T.; Uemura, S. J. Org. Chem. 2002, 67, 6718.

${ }^{2}$ Nishio, T.; Nakata, H.; Omote, Y. J. Heterocycl. Chem. 1986, 23, 1011.

${ }^{3}$ Pigge, F. C.; Ghasedi, F.; Zheng, Z.; Rath, N. P.; Nichols, G.; Chickos, J. S. J. Chem. Soc. Perkin Trans. 2; 2000;2458.

${ }^{4}$ Benedetti, F.; Fabrissin, S.; Fagotto, R.; Risaliti, A. Gazz. Chim. Ital. 1990, 120, 613.

${ }^{5}$ Hamashima, Y.; Hotta, D.; Sodeoka, M. J. Am. Chem. Soc. 2002, 124, 11240.
} 